\title{
I see what you mean
}

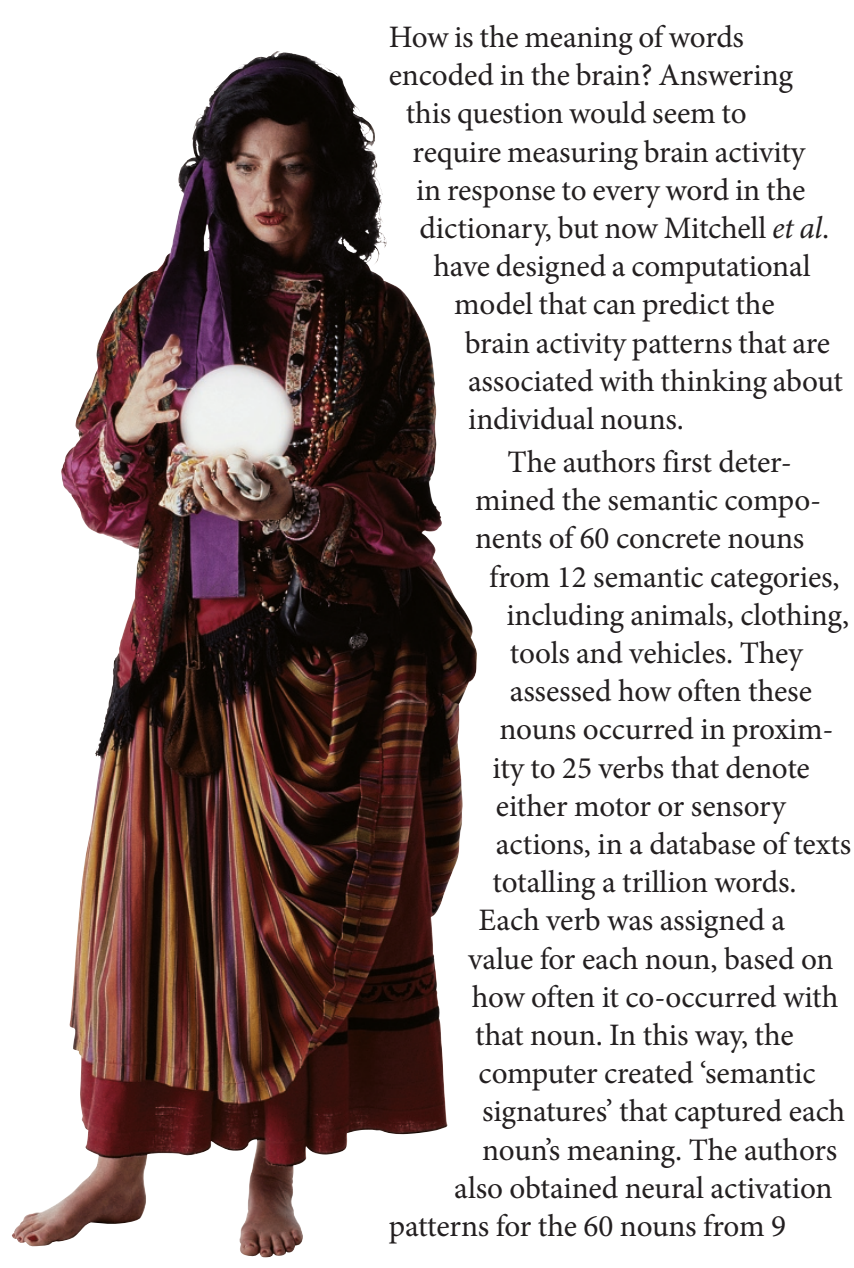

volunteers, who were asked to think about each of the nouns when they appeared on a screen inside a functional MRI (fMRI) scanner.

The authors then developed a computational model that learned to relate the neural and semantic signatures for each noun. The model was trained on 58 of the nouns and was then used to predict the neural signatures of the remaining two words from their semantic signatures. The predicted neural activation patterns matched the volunteers' actual fMRI scans for the two nouns with $77 \%$ accuracy. When the model was retrained using 59 of the nouns and then given the fMRI image associated with the noun that had been left out, it could pick the correct noun from a list of 1001 words with $72 \%$ rank accuracy.

Interestingly, when the authors assessed the neural activation that the model associated with each of the 25 verbs, they found that the brain areas that it linked to the verb 'eat' included the gustatory cortex, and that those that it linked to the verb 'run' included the part of the temporal lobe that is involved in the perception of biological motion. This suggests that among the brain areas that might encode a verb's meaning are areas that are activated when a person carries out the action that the verb signifies.

This model allows neuroscientists to estimate how any concrete noun is encoded in the brain, without carrying out thousands of brain scans. Extending the semantic signatures of nouns to include adjectives might further improve the model, in particular its ability to distinguish between the neural signatures of nouns from the same category (a task at which the model was not very successful). Refining the model might also allow it to predict the neural representation of more abstract concepts. Nevertheless, using only 25 verbs to semantically define nouns, the model produced remarkably accurate neural signatures, indicating that the neural representations of concrete nouns are at least partly based on the sensory and motor features of the object.

Leonie Welberg

ORIGINAL RESEARCH PAPER Mitchell, T. M. et al. Predicting human brain activity associated with the meanings of nouns. Science $\mathbf{3 2 0}$, 1191-1195 (2008) 